\title{
Uso do forno de microondas na determinação da umidade em diferentes tipos de solo
}

\section{Microwave oven use for soil moisture content determination in different soils}

\author{
Maria Hermínia Ferreira Tavares ${ }^{1 *}$; Décio Lopes Cardoso ${ }^{2}$; \\ Daniele Patrícia Gentelini³; Antonio Gabriel Filho²; Evandro André Konopatski ${ }^{3}$
}

\begin{abstract}
Resumo
No presente trabalho estudou-se a utilização do forno de microondas na determinação da umidade no solo, comparando-se os resultados com os valores fornecidos pelo método da estufa convencional, usando-se nove solos, com diferentes texturas. Os resultados obtidos por meio de qualquer um dos dois métodos não diferiram entre si. Quando se usou o método do microondas, as variações entre repetições diminuíram com o tamanho da amostra e com a percentagem de partículas finas no solo. A análise de regressão entre as variáveis tempo e umidade apresentou ajuste potencial do tipo $\mathrm{y}=\mathrm{kx}^{\mathrm{n}}$, com elevada correlação $\left(\mathrm{R}^{2}=0,9997\right)$ para todos os solos estudados. Os valores do expoente $\mathrm{n}$, todos próximos da unidade, indicaram que o mecanismo de retirada da água por microondas apresentou um comportamento quasi-linear em função do tempo e que os teores de umidade inicial e a massa de solo não influenciaram o processo. Os valores de $\mathrm{k}$ nas equações de regressão indicaram que o processo de secagem no forno de microondas tem maior efeito sobre solos argilosos em relação àqueles arenosos. $\mathrm{O}$ forno de microondas pode ser empregado na determinação da umidade em solos, ocasionando significativa economia de tempo.
\end{abstract}

Palavras-chave: Campo eletromagnético, secagem em estufa, teor de umidade

\begin{abstract}
In the present research the use of a microwave oven for the soil moisture content determination was analyzed, comparing the results with the values given by the conventional oven drying, using nine soils, with different textures. The results obtained by either method did not show appreciable differences for the soil samples. When the microwave oven was used, the variation among replicates decreased with the sample size and with the fine particles percentage in the soil. The regression analysis showed that a power law, $\mathrm{y}=\mathrm{kx}^{\mathrm{n}}$, adjusted the date with a large correlation $\left(\mathrm{R}^{2}=0.9997\right)$ for all the soils. The $\mathrm{n}$ exponent values, near to the unity, indicated that the water removal mechanism showed a behavior near to the linearity in function of the time and that neither the initial water content nor the soil mass influenced the process. The $\mathrm{k}$ values in the regression equations showed that the process acts more intensively on clayey soils than on sandy ones. The experimental results allowed to conclude that the microwave oven may be used as an alternative to soil content measurement, resulting in time economy.
\end{abstract}

Key words: Electromagnetic field, oven drying, soil moisture content

\footnotetext{
${ }_{1}$ Docente do Programa de Pós-Graduação em Engenharia Agrícola, Universidade Estadual do Oeste do Paraná, Cascavel, PR. E-mail: mhstavar@certto.com.br

2 Docentes do Programa de Pós-Graduação em Engenharia Agrícola, Universidade Estadual do Oeste do Paraná, Cascavel, PR.

${ }^{3}$ Mestre em Engenharia Agrícola.

* Autor para correspondência
} 


\section{Introdução}

O uso de estufa é um método consagrado para a estimativa da umidade em solos, segundo a EMPRESA BRASILEIRA DE PESQUISA AGROPECUÁRIA (1997). Entretanto, quando se pretende rapidez em se conhecer a umidade, tal método não é eficiente, pois até mesmo o intervalo de tempo adequado para se realizar a secagem é controverso. Conforme Benke e Kearfott (1999), a dependência do teor de umidade com o tempo de secagem da amostra em estufa pode ser expressa através de uma função Gaussiana com dois parâmetros e a umidade inicial das amostras não afeta o resultado final. Diferentes alternativas ao emprego da estufa têm sido propostas, sendo uma delas a aplicação de microondas. Miller, Smith e Biggar (1974) propuseram o uso do forno de microondas comercial, o que diminui sobremaneira o tempo de secagem, o qual é função do número de amostras, do tamanho e potência do forno, do teor de umidade inicial, da massa da amostra e do tipo de solo.

O processo de secagem do solo através do emprego da radiação eletromagnética baseiase no fato das moléculas de água serem dipolos elétricos naturais, os quais sofrem rotação quando expostos a um campo eletromagnético. Segundo Aguilar (2001), o atrito molecular resultante gera calor instantânea e uniformemente na amostra de solo, reduzindo a umidade. Já o comportamento de materiais ferromagnéticos na presença de campos elétricos externos, tais como o campo magnético de uma onda eletromagnética do tipo microonda (GUIMARÃES, 1998), é determinado pela origem de seus dipolos magnéticos e pela natureza da interação entre eles. Assim, solos com altos teores de Fe apresentam comportamento peculiar, quando submetidos à radiação eletromagnética.

Embora vários trabalhos mostrem que a secagem de solos em forno de microondas pode ser empregada com sucesso, com relação a outras análises os resultados são diferentes: por exemplo, Tomé e Dechen (1995) mostraram que a irradiação por microondas pode acarretar mudanças nos resultados de análises químicas e mineralógicas.

O presente trabalho foi realizado com o objetivo de se verificar a possibilidade da adoção do forno de microondas em determinações rotineiras da umidade de solos, em amostras de diferentes composições granulométricas.

\section{Material e Métodos}

Os solos utilizados no experimento estão apresentados na Tabela 1, seguindo a classificação da EMPRESA BRASILEIRA DE PESQUISA AGROPECUÁRIA (2006), com exceção do solo de número 8 , o qual não se enquadra na classificação.

Tabela 1. Classificação e procedência dos solos estudados.

\begin{tabular}{ccc}
\hline SOLO & CLASSIFICAÇÃO & PROCEDÊNCIA \\
\hline 1 & Latossolo Vermelho Distroférrico típico & Cascavel - PR \\
2 & Argissolo Vermelho-Amarelo eutrófico & Umuarama - PR \\
3 & Latossolo Vermelho Distrófico & Umuarama - PR \\
4 & Argissolo Amarelo Distrófico & Curitiba - PR \\
5 & Argissolo Amarelo Distrófico arênico & Ilha do Mel - PR \\
6 & Cambissolo Háplico & Matinhos - PR \\
7 & Neossolo Quartzarênico Hidromorfico & Florianópolis - SC \\
8 & Argilomineral Caulinita & Monte Pascoal - BA \\
9 & Neossolo Quartzarênico & Guaira - PR \\
\hline
\end{tabular}


A caulinita foi incluída como solo na Tabela 1 por ser um argilomineral, mineral secundário formado a partir da síntese de minerais primários existentes nas rochas. Mais especificamente, a caulinita é formada por uma seqüência de reações de hidrólise entre o mineral primário feldspato e os íons dissociados $\mathrm{H}^{+}$e $\mathrm{OH}^{-}$da água, o que ocorre em ambiente de formação de solos, ou seja, com intemperismo. Nesse contexto, é razoável classificar tal material como solo.

As curvas de distribuição granulométrica dos solos estudados são apresentadas na Figura 1.

Na Tabela 2 são mostrados atributos físicos e químicos do SOLO 1, o qual apresentou os maiores teores de argila e de ferro entre os nove solos estudados.

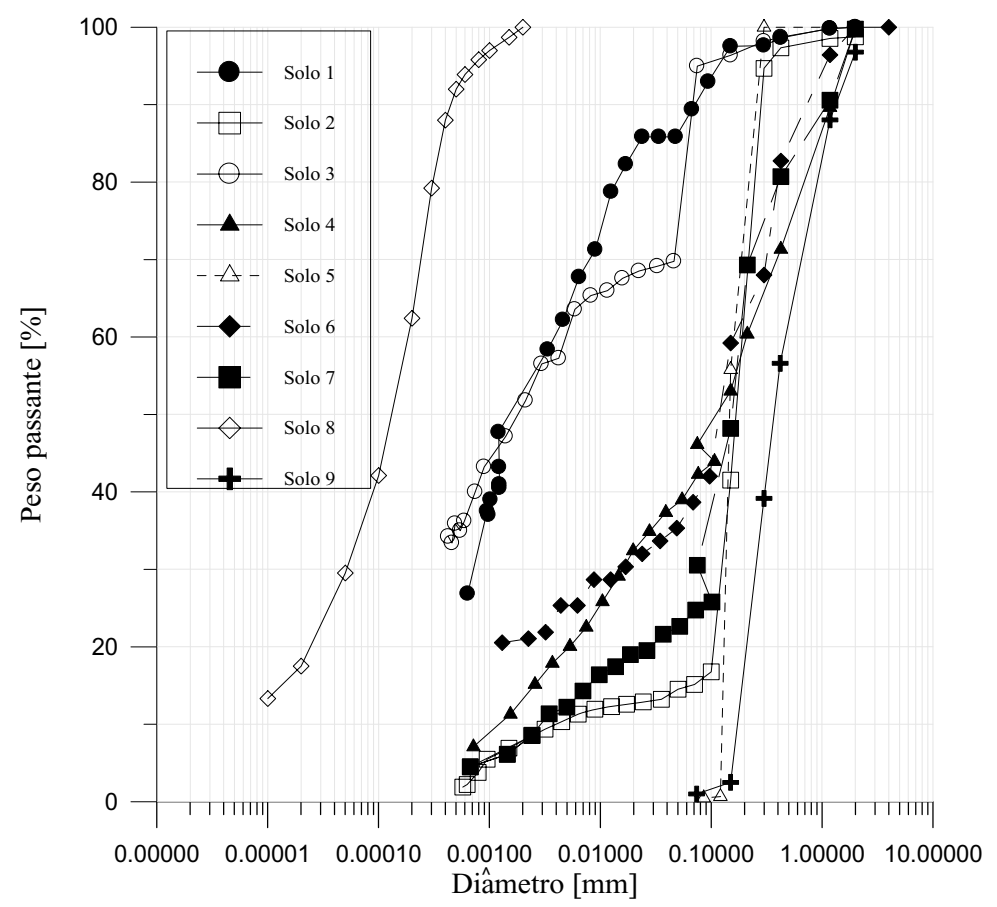

Figura 1. Curvas de distribuição granulométrica dos solos estudados.

Tabela 2. Atributos físicos e químicos do SOLO 1 (camada de 5,0 a 25,0 cm).

\begin{tabular}{cccc}
\hline Atributo & Valor & Atributo & Valor \\
\hline Areia & $3,0 \%$ & $\mathrm{pH}\left(\mathrm{CaCl}_{2}\right)$ & 5,80 \\
Silte & $27,9 \%$ & $\mathrm{C}$ & $23,22 \mathrm{mgdm}^{-3}$ \\
Argila & $69,1 \%$ & $\mathrm{P}$ & $5,70 \mathrm{mgdm}^{-3}$ \\
Densidade do solo & $1,31 \mathrm{Mgm}^{-3}$ & $\mathrm{Fe}$ & $68,10 \mathrm{mgdm}^{-3}$ \\
Densidade de partículas & $2,92 \mathrm{Mgm}^{-3}$ & $\mathrm{~K}^{+}$ & $0,14 \mathrm{cmoldm}^{-3}$ \\
Porosidade Total & $55,0 \%$ & $\mathrm{Ca}^{2+}$ & $7,73 \mathrm{cmoldm}^{-3}$ \\
Macroporosidade & $10,4 \%$ & $\mathrm{Mg}^{2+}$ & $4,60 \mathrm{cmoldm}^{-3}$ \\
Microporosidade & $44,6 \%$ & $\mathrm{H}+\mathrm{Al}^{3+}$ & $4,61 \mathrm{cmoldm}^{-3}$ \\
Umidade volumétrica na capacidade & $0,405 \mathrm{~m}^{3} \mathrm{~m}^{-3}$ & & \\
de campo & & & \\
Umidade volumétrica no ponto de & $0,253 \mathrm{~m}^{3} \mathrm{~m}^{-3}$ & & \\
murcha permanente & & &
\end{tabular}


As amostras foram secas ao ar, peneiradas com peneira de 2,00 $\mathrm{mm}$ de abertura, sendo em seguida molhadas com água destilada e homogeneizadas. $\mathrm{O}$ solo homogeneizado foi acondicionado em saco plástico, o qual foi hermeticamente fechado por 24 horas. Decorrido o tempo, foram retiradas três cápsulas para determinação do teor médio de umidade em estufa a $105^{\circ} \mathrm{C}$ por 24 horas, para efeito de comparação, segundo EMPRESA BRASILEIRA DE PESQUISA AGROPECUÁRIA (1997). A determinação do teor médio de umidade por microondas foi conduzida em forno BRASTEMP modelo BMP28EHA, com tensão de alimentação de 120 $\mathrm{V}(60 \mathrm{~Hz})$, freqüência de microondas $2,450 \mathrm{MHz}$, segundo BRASTEMP (1991). As determinações foram efetuadas com nível de potência alta (10), com as amostras de solo acondicionadas em cadinhos de porcelana. Foram empregados os seguintes valores de massa: 20, 40 e 60 g. Inicialmente, as amostras foram submetidas a um tempo de 120 segundos no forno de microondas. Em seguida, os cadinhos foram retirados, aguardou-se o resfriamento em dessecador e fez-se a pesagem em balança eletrônica com precisão de $0,01 \mathrm{~g}$. Este procedimento foi repetido de 120 em 120 segundos, até a estabilização da massa da amostra, segundo TEXAS DEPARTMENT OF TRANSPORTATION (2002).

Para cada massa, foram empregadas cinco repetições no processo de secagem no forno de microondas, a partir das quais a porcentagem de umidade média foi calculada. Posteriormente, os valores médios de umidade foram submetidos ao teste de comparação de médias pelo teste de Tukey a 5\%, utilizando-se o programa Minitab versão 14.0 (MINITAB, 2003). Os valores médios de umidade, determinados através do uso do forno de microondas, também foram empregados para ajustar as equações de regressão, relacionando o tempo de secagem com a umidade gravimétrica. As análises de regressão foram realizadas com o intervalo de confiança igual a 95\%, escolhendo-se a equação de melhor ajuste de acordo com o maior coeficiente de determinação.

\section{Resultados e Discussão}

Os resultados das comparações de médias dos valores de umidade gravimétrica estão nas Tabelas 3,4 e 5 , as quais mostram que o tempo de secagem de 240 segundos foi suficiente para a estabilização de massa em todos os solos estudados.

Tabela 3. Valores de umidade gravimétrica (\%) obtidos através do forno de microondas para a massa de $20 \mathrm{~g}$, nos três tempos empregados.

\begin{tabular}{cccc}
\hline Solo & $\mathbf{1 2 0} \mathbf{s}$ & $\mathbf{2 4 0} \mathbf{s}$ & $\mathbf{3 6 0} \mathbf{s}$ \\
\hline 1 & $45,93 \mathrm{~b}$ & $47,84 \mathrm{a}$ & $47,98 \mathrm{a}$ \\
2 & $19,69 \mathrm{~b}$ & $21,93 \mathrm{a}$ & $22,08 \mathrm{a}$ \\
3 & $19,19 \mathrm{~b}$ & $19,48 \mathrm{a}$ & $19,54 \mathrm{a}$ \\
4 & $32,39 \mathrm{a}$ & $33,57 \mathrm{a}$ & $33,64 \mathrm{a}$ \\
5 & $22,60 \mathrm{a}$ & $22,60 \mathrm{a}$ & $22,60 \mathrm{a}$ \\
6 & $27,55 \mathrm{~b}$ & $35,73 \mathrm{a}$ & $37,34 \mathrm{a}$ \\
7 & $22,58 \mathrm{a}$ & $22,69 \mathrm{a}$ & $22,69 \mathrm{a}$ \\
8 & $55,39 \mathrm{~b}$ & $56,68 \mathrm{a}$ & $56,83 \mathrm{a}$ \\
9 & $19,64 \mathrm{a}$ & $22,13 \mathrm{a}$ & $22,13 \mathrm{a}$ \\
\hline
\end{tabular}

Valores seguidos da mesma letra, na linha, não diferem entre si, pelo teste de Tukey a $5 \%$. 
Tabela 4. Valores de umidade gravimétrica (\%) obtidos através do forno de microondas para a massa de $40 \mathrm{~g}$, nos três tempos empregados.

\begin{tabular}{cccc}
\hline Solo & $\mathbf{1 2 0} \mathbf{s}$ & $\mathbf{2 4 0} \mathbf{s}$ & $\mathbf{3 6 0} \mathbf{s}$ \\
\hline 1 & $44,80 \mathrm{~b}$ & $48,50 \mathrm{a}$ & $49,11 \mathrm{a}$ \\
2 & $19,44 \mathrm{~b}$ & $21,65 \mathrm{a}$ & $21,65 \mathrm{a}$ \\
3 & $18,53 \mathrm{~b}$ & $19,49 \mathrm{a}$ & $19,49 \mathrm{a}$ \\
4 & $34,38 \mathrm{a}$ & $34,85 \mathrm{a}$ & $34,88 \mathrm{a}$ \\
5 & $23,05 \mathrm{a}$ & $23,05 \mathrm{a}$ & $23,08 \mathrm{a}$ \\
6 & $25,49 \mathrm{~b}$ & $35,86 \mathrm{a}$ & $36,36 \mathrm{a}$ \\
7 & $22,32 \mathrm{a}$ & $22,58 \mathrm{a}$ & $22,61 \mathrm{a}$ \\
8 & $53,85 \mathrm{~b}$ & $56,89 \mathrm{a}$ & $56,89 \mathrm{a}$ \\
9 & $15,77 \mathrm{~b}$ & $21,45 \mathrm{a}$ & $22,05 \mathrm{a}$ \\
\hline
\end{tabular}

Valores seguidos da mesma letra, na linha, não diferem entre si, pelo teste de Tukey a 5\%.

Tabela 5. Valores de umidade gravimétrica (\%) obtidos através do forno de microondas para a massa de $60 \mathrm{~g}$, nos três tempos empregados.

\begin{tabular}{cccc}
\hline Solo & $\mathbf{1 2 0} \mathbf{s}$ & $\mathbf{2 4 0} \mathbf{s}$ & $\mathbf{3 6 0} \mathbf{s}$ \\
\hline 1 & $41,34 \mathrm{~b}$ & $48,87 \mathrm{a}$ & $50,31 \mathrm{a}$ \\
2 & $16,80 \mathrm{~b}$ & $21,64 \mathrm{a}$ & $21,70 \mathrm{a}$ \\
3 & $17,47 \mathrm{~b}$ & $18,73 \mathrm{a}$ & $18,77 \mathrm{a}$ \\
4 & $33,47 \mathrm{a}$ & $34,30 \mathrm{a}$ & $34,30 \mathrm{a}$ \\
5 & $22,63 \mathrm{a}$ & $22,65 \mathrm{a}$ & $22,65 \mathrm{a}$ \\
6 & $30,03 \mathrm{~b}$ & $36,38 \mathrm{a}$ & $36,22 \mathrm{a}$ \\
7 & $21,53 \mathrm{a}$ & $22,22 \mathrm{a}$ & $22,24 \mathrm{a}$ \\
8 & $46,72 \mathrm{~b}$ & $56,27 \mathrm{a}$ & $56,45 \mathrm{a}$ \\
9 & $17,26 \mathrm{~b}$ & $21,29 \mathrm{a}$ & $21,63 \mathrm{a}$ \\
\hline
\end{tabular}

Valores seguidos da mesma letra, na linha, não diferem entre si, pelo teste de Tukey a $5 \%$.

As Figuras 2 a 10 apresentam o comportamento da umidade média em função do tempo, para os

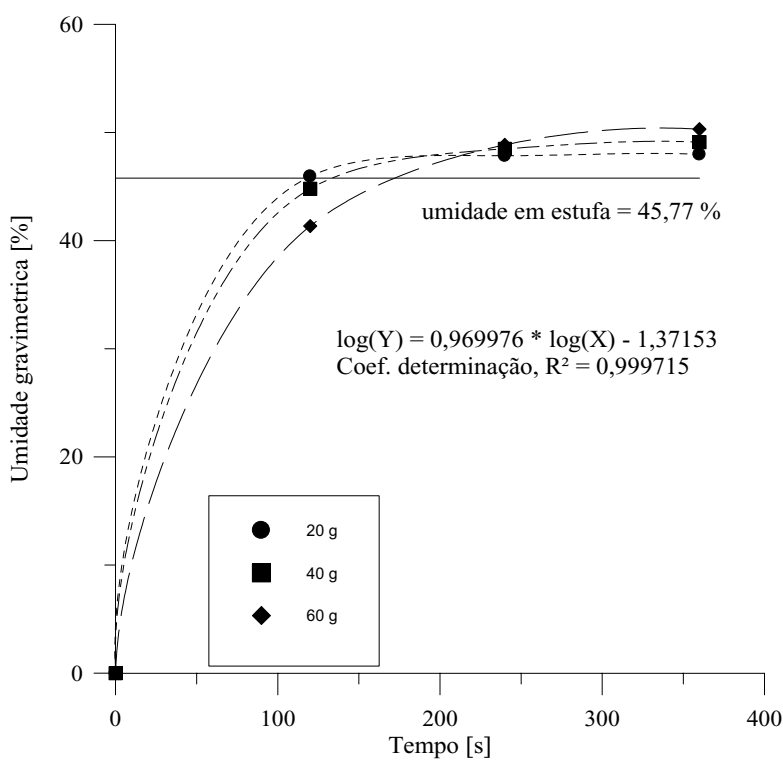

Figura 2. Comportamento da umidade média em função do tempo e da massa da amostra para o SOLO 1. nove tipos de solo, com secagem no forno de microondas.

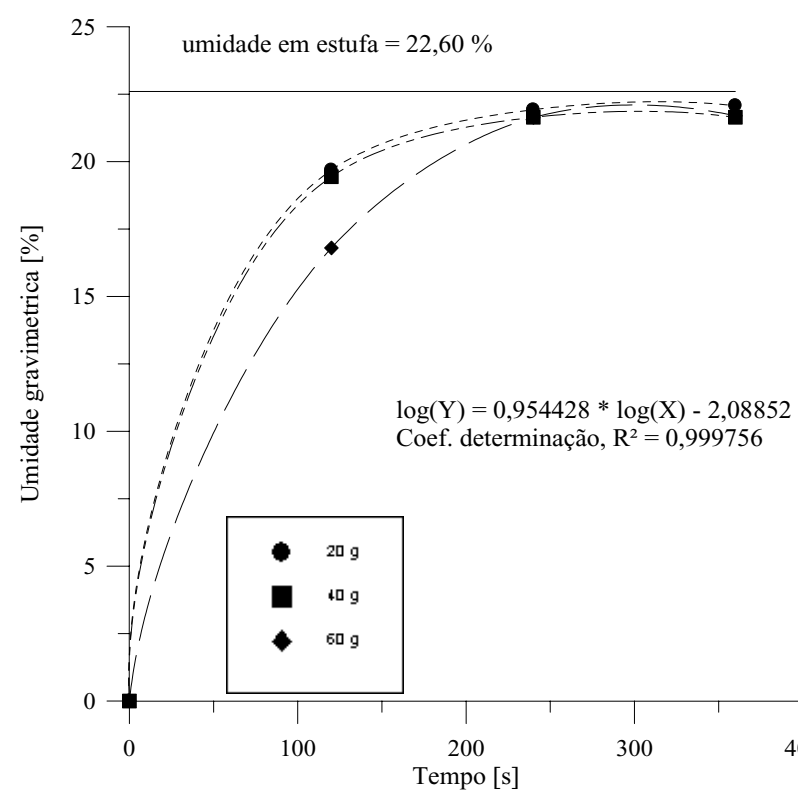

Figura 3. Comportamento da umidade média em função do tempo e da massa da amostra para o SOLO 2. 


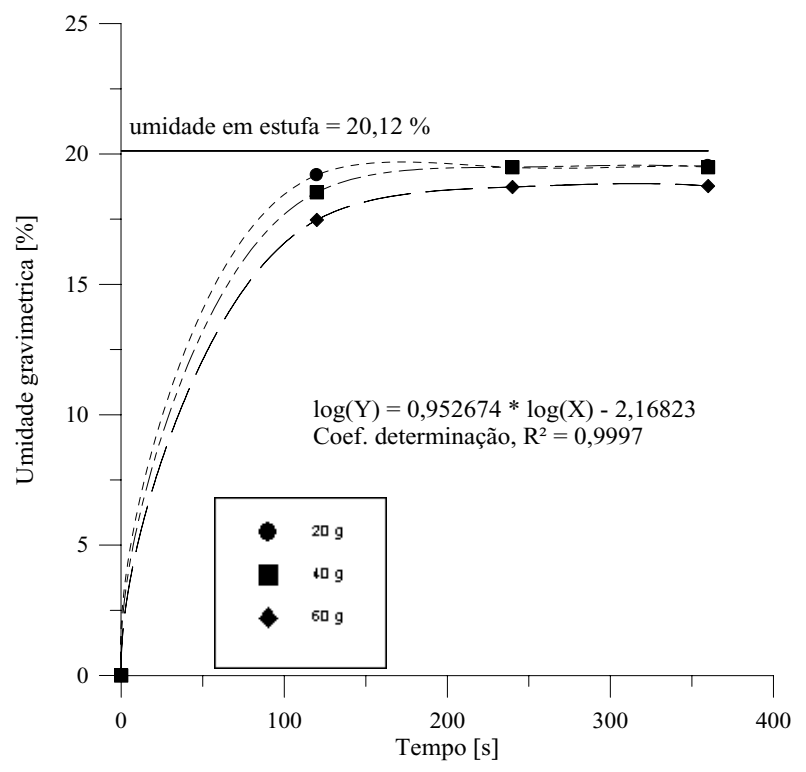

Figura 4. Comportamento da umidade média em função do tempo e da massa da amostra para o SOLO 3.

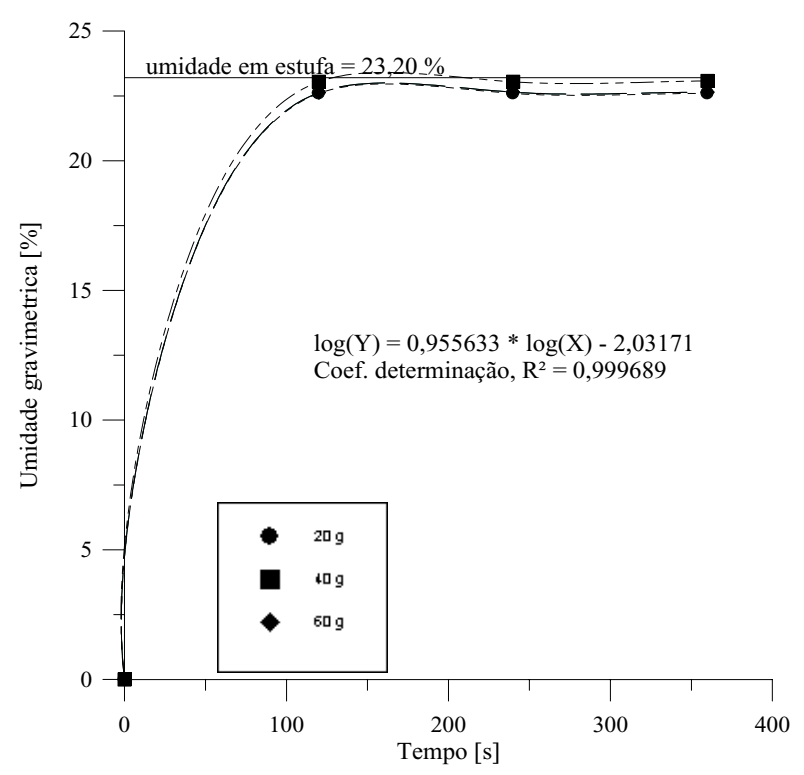

Figura 6. Comportamento da umidade média em função do tempo e da massa da amostra para o SOLO 5.

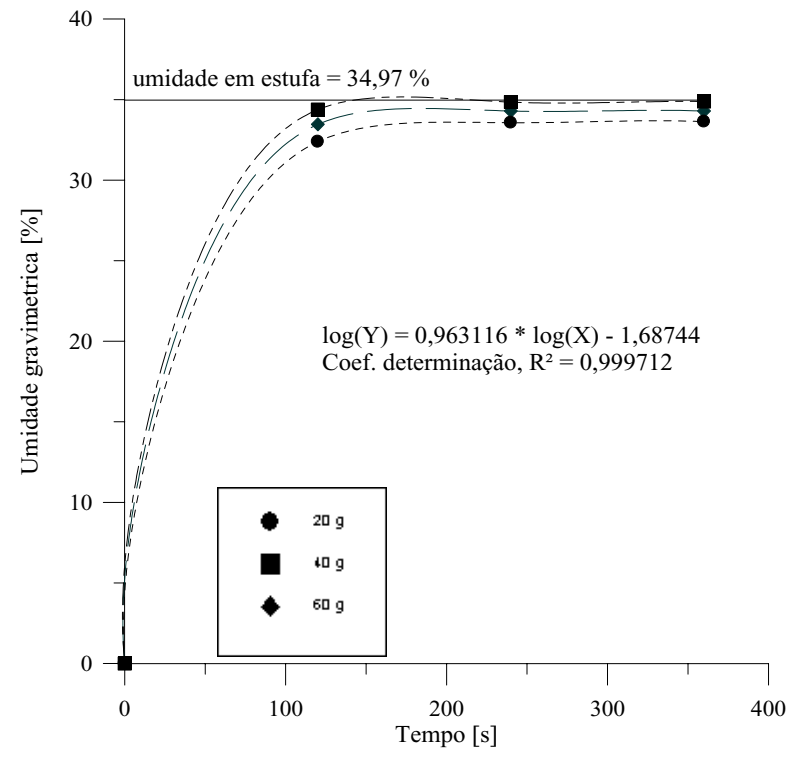

Figura 5. Comportamento da umidade média em função do tempo e da massa da amostra para o SOLO 4.

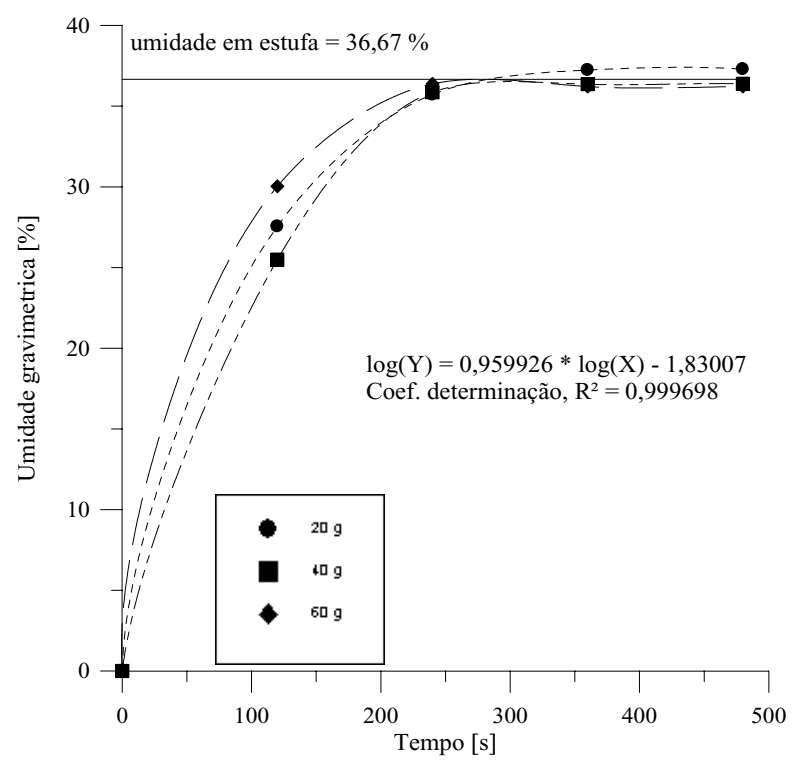

Figura 7. Comportamento da umidade média em função do tempo e da massa da amostra para o SOLO 6. 


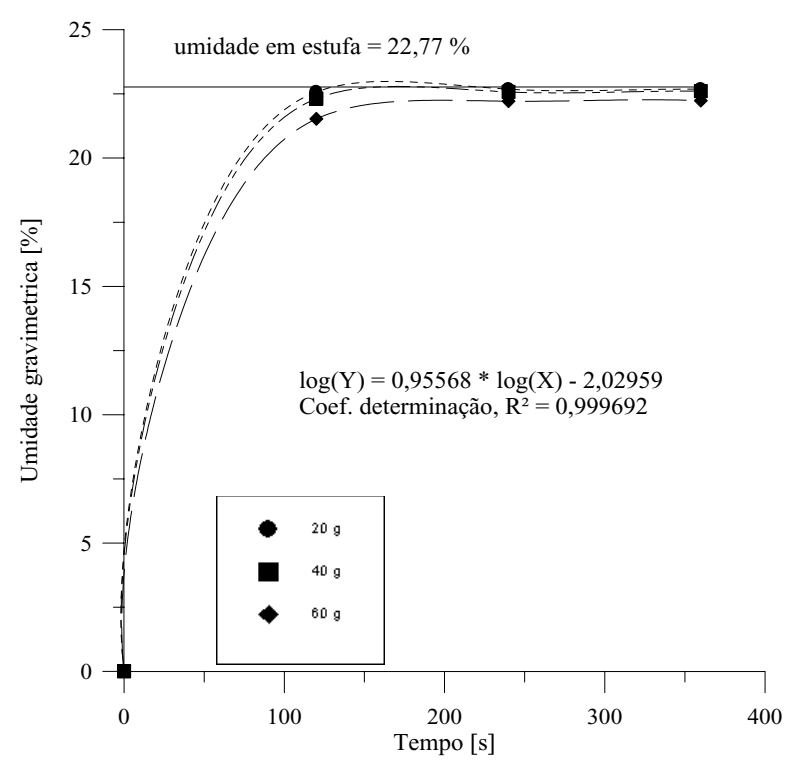

Figura 8. Comportamento da umidade média em função do tempo e da massa da amostra para o SOLO 7.

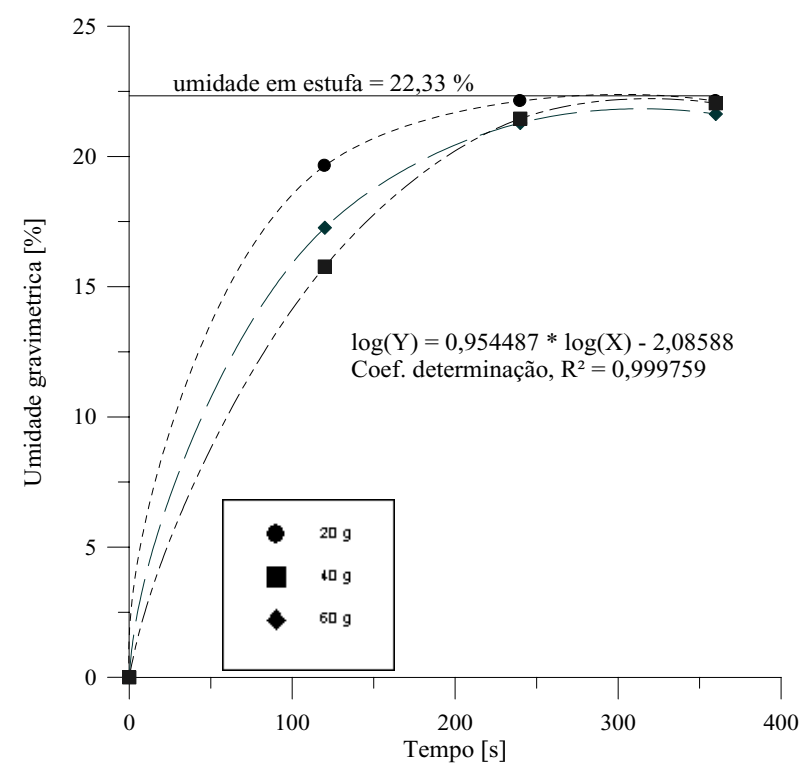

Figura 10. Comportamento da umidade média em função do tempo e da massa da amostra para o SOLO 9.

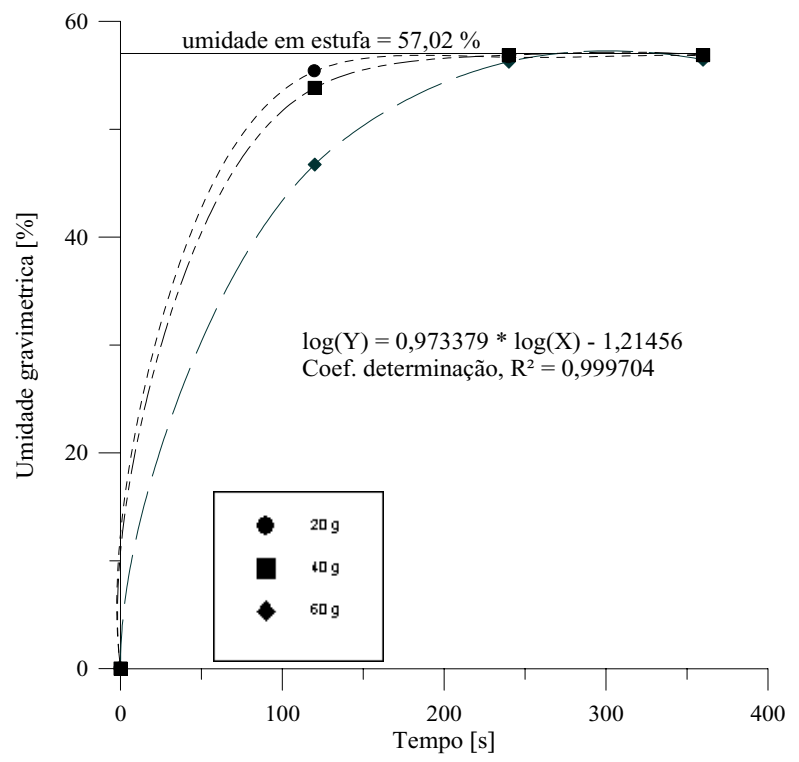

Figura 9. Comportamento da umidade média em função do tempo e da massa da amostra para o SOLO 8.

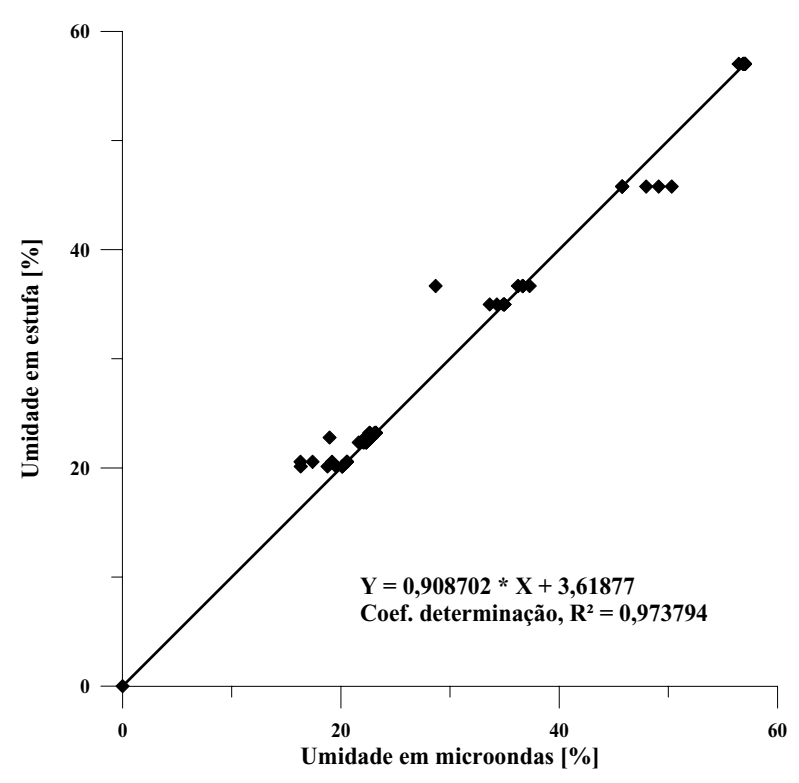

Figura 11. Relação entre o teor de umidade de estufa e o teor de umidade de microondas. 
Os elevados coeficientes de determinação obtidos na análise de regressão para todos os solos estudados $\left(\mathrm{R}^{2}=0,99997\right)$, permitem discutir os resultados a partir dos parâmetros da regressão. Observa-se, por exemplo, que os expoentes das equações da regressão variam de 0,95 a 0,97 , muito próximo de 1 . Isto implica que o mecanismo de retirada da água por microondas apresenta um comportamento quasi-linear em função do tempo. Além disso, o expoente próximo de 1 indica que o teor de umidade inicial não influencia o processo, bem como a massa da amostra, confirmando os resultados de Benke e Kearfott (1999). A independência dos resultados com relação à massa inicial da amostra também está de acordo com os resultados obtidos por Chieppe (1993). As constantes aditivas nas equações de regressão, as quais se mostraram mais elevadas para solos mais argilosos, indicam que o processo tem um maior efeito sobre esses, em relação aos solos arenosos.

Na Figura 11 são apresentados os teores de umidade finais no forno de microondas e na estufa para as três massas testadas, para todos os tipos de solos. A reta teórica apresentada tem inclinação de $45^{\circ}$, demodo quea distância dos pontos experimentais até a mesma fornece a dispersão dos dados. $\mathrm{O}$ ajuste obtido mostra que se pode fazer a estimativa do teor de umidade na estufa, conhecendo-se o teor de umidade obtido no forno de microondas.

Analisando as curvas apresentadas anteriormente, verifica-se que a remoção de umidade no SOLO 1 foi menor no forno de microondas do que na estufa, diferentemente do que ocorreu com os demais solos. Tal resultado pode estar associado ao alto teor de ferro presente no SOLO 1, absorvendo energia das microondas, estabelecendo diferentes gradientes térmicos dentro do sistema a ser aquecido pela radiação, conforme descreve Aguilar (2001). Ainda um outro aspecto a ser observado é a tendência precoce de estabilização da umidade do solo arenoso, confirmando Miller, Smith e Biggar (1974).
Observando-se os resultados, é possível verificar a aplicabilidade da técnica de microondas na determinação do teor de umidade do solo, com a redução do tempo necessário para o trabalho, pois, nos nove solos analisados, o tempo médio para a completa depleção da umidade foi de 240 segundos.

\section{Conclusões}

O tempo de secagem de 240 segundos foi suficiente para a estabilização de massa em todos os solos estudados.

O processo de secagem no forno de microondas tem maior efeito sobre solos argilosos em relação àqueles arenosos.

O teor de umidade no solo pode ser estimado de forma rápida, a partir dos valores de umidade obtidos no forno de microondas.

\section{Referências}

AGUILAR, J. A. G. Procesamiento de materiales por medio de microondas em la FIME. Ingenierías, Nuevo León, v. 4, n. 13, n., p. 32-39, 2001.

BENKE, R. R.; KEARFOTT, K. J. Soil sample moisture content as a function of time during oven drying for gamma-ray spectroscopic measurements. Nuclear Instruments and Methods in Physics Research Section A: Accelerators, Spectrometers, Detectors and Associated Equipment, Amsterdam, v. 422, n. 3, p. 817-819, 1999.

BRASTEMP. Microondas: manual do consumidor. São Bernardo do Campo: BRASTEMP - Depto. de Relações com o Consumidor, 1991.

CHIEPPE, J. B. Estudo e comparação de diferentes métodos de determinação de umidade do solo em laboratório. 1993. Dissertação (Mestrado em Agronomia) - Universidade Estadual Paulista Júlio de Mesquita Filho, Botucatu.

EMPRESA BRASILEIRA DE PESQUISA AGROPECUÁRIA - EMBRAPA. Manual de métodos de análise de solos. 2.ed. Rio de Janeiro: Centro Nacional de Pesquisa de Solos, 1997. 
Sistema brasileiro de classificação de solos. 2.ed. Rio de Janeiro: Centro Nacional de Pesquisas de Solos, 2006.

GUIMARÃES, A. P. Magnetism and magnetic resonance in solids. New York: John Wiley \& Sons, 1998.

MILLER, R. J.; SMITH, R. B.; BIGGAR, J.W. Soil water content: microwave oven method. Proceedings Soil Science Society of America, Madison, v. 38, n. 3, p. 535-537, 1974.
MINITAB. Minitab for Windows: Version 14.0 PA. State College: Minitab Statistical Software, 2003.

TEXAS DEPARTMENT OF TRANSPORTATION TDT. Manual of testing procedures. Texas: TDT, 2002.

TOMÉ, J. B.; DECHEN, A. R. Microwave ovendrying of soil samples for chemical testing in Brazil. Communications in soil science and plant analysis, New York, v. 26, n. 3-4, p. 515-529, 1995. 
